\title{
Qualidade da atenção pré-natal na rede básica de saúde do Brasil: indicadores e desigualdades sociais
}

\section{Quality of prenatal services in primary healthcare in Brazil: indicators and social inequalities}

\section{Calidad de la atención prenatal en la red básica de salud de Brasil: indicadores y desigualdades sociales}

Elaine Tomasi 1

Pedro Agner Aguiar Fernandes 1

Talita Fischer 1

Fernando Carlos Vinholes Siqueira 2

Denise Silva da Silveira 1

Elaine Thumé 3

Suele Manjourany Silva Duro 3

Mirelle de Oliveira Saes 4

Bruno Pereira Nunes 3

Anaclaudia Gastal Fassa 1

Luiz Augusto Facchini 1

doi: 10.1590/0102-311X00195815

\section{Resumo}

O objetivo foi descrever indicadores de qualidade da atenção pré-natal no Brasil no âmbito do Programa de Melhoria do Acesso e da Qualidade (PMAQ-AB). Foram analisados número de consultas, situação vacinal, prescrição de sulfato ferroso, exame físico, orientações e exames complementares, com base no que se construiu como um indicador sintético de qualidade. Os dados foram coletados em 2012/2013 por meio de entrevistas realizadas por Avaliadores Externos do PMAQ-AB às 6.125 usuárias que fizeram seu último pré-natal nas unidades de saúde da família. Durante o pré-natal, 89\% fizeram seis ou mais consultas, mais de 95\% atualizaram a vacina antitetânica e receberam prescrição de sulfato ferroso, 24\% referiram ter recebido todos os procedimentos de exame físico, 60\% receberam todas as orientações e $69 \%$ realizaram todos os exames complementares. Apenas $15 \%$ das entrevistadas receberam atenção pré-natal adequada, considerando-se todas as ações preconizadas, sendo significativamente maior a proporção de completude da atenção em gestantes com mais idade, de maior renda, na Região Sudeste, nos municípios com mais de 300 mil habitantes e com IDH no quartil superior. Persistem desigualdades sociais e individuais que podem ser objeto de ações de qualificação dos processos de trabalho das equipes.

Cuidado Pré-Natal; Atenção Primária à Saúde; Qualidade da Assistência à Saúde; Desigualdades em Saúde

\author{
Correspondência \\ E. Tomasi \\ Departamento de Medicina Social, Faculdade de Medicina, \\ Universidade Federal de Pelotas. \\ Av. Duque de Caxias 250, Pelotas, RS 96030-001, Brasil. \\ tomasiet@gmail.com \\ 1 Faculdade de Medicina, Universidade Federal de Pelotas, \\ Pelotas, Brasil. \\ 2 Escola Superior de Educação Física, Universidade Federal de \\ Pelotas, Pelotas, Brasil. \\ 3 Faculdade de Enfermagem, Universidade Federal de Pelotas, \\ Pelotas, Brasil. \\ 4 Programa de Pós-graduação em Ciências da Saúde, \\ Universidade Federal do Rio Grande, Rio Grande, Brasil.
}




\section{Introdução}

A manutenção e a melhoria da saúde materno-infantil são alguns dos objetivos definidos pelo Ministério da Saúde e, para isto, é essencial a atenção pré-natal e puerperal, cuja responsabilidade é do Sistema Único de Saúde (SUS) ${ }^{1}$. No âmbito da Rede Cegonha, a atenção à mulher durante a gravidez e pós-parto preconiza ações de prevenção e promoção da saúde, além de diagnóstico e tratamento adequado dos problemas que ocorrem neste período 2 .

Uma atenção pré-natal de qualidade é capaz de diminuir a morbidade e a mortalidade materno-infantil 3,4,5,6, uma vez que a identificação do risco gestacional pelo profissional permite a orientação e os encaminhamentos adequados em cada momento da gravidez 7,8. Em 2014, cerca de 40\% dos 10.446 óbitos infantis e neonatais evitáveis ocorridos no Brasil estavam relacionados à inadequação da atenção à gestação (Sistema de Informações sobre Mortalidade. Painel de monitoramento da mortalidade infantil e fetal. http://svs.aids.gov.br/dashboard/mortalidade/infantil.show.mtw, acessado em 12/Ago/2015).

Apesar de quase a totalidade das gestantes brasileiras fazer pelo menos alguma consulta de prénatal, a proporção que realizou seis ou mais consultas foi de 73\% em 2012 (Departamento de Informática do SUS. Indicadores e dados básicos - Brasil, 2012. IDB-2012. http://tabnet.datasus.gov.br/ cgi/idb2012/matriz.htm, acessado em 14/Set/2015) e este percentual foi menor em mulheres de nível econômico mais baixo, nas mais jovens e naquelas menos escolarizadas 9,10. Também o início precoce do acompanhamento pré-natal atinge apenas três quartos das mulheres, sendo menor para as mais jovens, negras e das regiões Norte e Nordeste do país 10 . Outros aspectos da atenção têm sido descritos com índices preocupantes, como a qualidade dos registros 11, a falta de solicitação de exames laboratoriais 12 , de exames clínico-obstétricos padronizados e de orientações sobre a gestação, como complicações da gravidez, preparação para o parto, aleitamento materno e cuidado com o recém-nascido 13 , apontando de forma consistente as desigualdades socioeconômicas na atenção pré-natal 10,14,15,16.

Com a ampliação da Estratégia Saúde da Família (ESF), que alcançou em julho de 2015 mais de 40 mil equipes e uma cobertura populacional de cerca de 65\%, a partir de 2011 o Ministério da Saúde implantou o Programa de Melhoria do Acesso e da Qualidade da Atenção Básica (PMAQ-AB). O PMAQ-AB objetiva induzir a ampliação do acesso da população aos serviços, a melhoria das condições de trabalho e, principalmente, da qualidade da atenção ${ }^{17}$. As equipes são incluídas no programa mediante adesão da gestão municipal e, após processo de avaliação externa, passam a ser certificadas progressivamente e contam com repasse de recursos em função de seu desempenho. Nesse contexto, um dos aspectos avaliados é a oferta de uma atenção pré-natal de qualidade 18.

O objetivo deste trabalho foi descrever a qualidade da atenção pré-natal no Brasil no âmbito da avaliação externa do PMAQ-AB, incluindo número de consultas, situação vacinal, prescrição de sulfato ferroso, procedimentos de exame físico, orientações fornecidas e exames complementares realizados, e investigar diferenciais sociodemográficos e contextuais a serem considerados na avaliação e planejamento desta política de saúde.

\section{Métodos}

Como parte da avaliação externa do primeiro ciclo do PMAQ-AB, 41 instituições brasileiras de ensino superior, lideradas pela Fundação Osvaldo Cruz (Fiocruz), Universidade Federal da Bahia (UFBA), Universidade Federal de Minas Gerais (UFMG), Universidade Federal de Pelotas (UFPel), Universidade Federal do Rio Grande do Sul (UFRGS) e Universidade Federal do Rio Grande do Norte (UFRN) realizaram um estudo transversal. Os dados foram coletados entre junho de 2012 e março de 2013 e o trabalho de campo envolveu cerca de mil entrevistadores e supervisores em todas as Unidades da Federação (UF), capacitados pelas instituições líder.

Foi realizado o censo de todas as unidades básicas de saúde (UBS) registradas no Cadastro Nacional de Estabelecimentos de Saúde, localizadas nas zonas urbana e rural dos 5.565 municípios do Brasil, além do Distrito Federal (Instituto Brasileiro de Geografia e Estatística. Censo demográfico de 2010. http://www.ibge.gov.br). O instrumento de coleta de dados da avaliação externa do PMAQ-AB 
foi composto por três módulos: Módulo I - observação na UBS; Módulo II - entrevista com um profissional sobre o processo de trabalho da equipe de atenção básica; Módulo III - entrevista com usuário na UBS 19.

As informações sobre a atenção pré-natal utilizadas neste estudo foram obtidas por meio da aplicação do Módulo III para mulheres adscritas à equipe, com 18 anos ou mais, presentes na UBS por ocasião da avaliação e que responderam afirmativamente a quatro perguntas: (1) "A Sra. já esteve grávida?"; (2) Se sim: "A Sra. tem filhos menores de dois anos?"; (3) Se sim: "A Sra. fez pré-natal na última gravidez?"; (4) Se sim: "O pré-natal foi realizado nesta unidade de saúde?".

Foram também incluídas 227 mulheres (3,8\%) com menos de 18 anos que atendiam aos demais critérios de inclusão por estarem acompanhadas de responsável que assinou o Termo de Consentimento Livre e Esclarecido.

Um total de seis desfechos foram selecionados e analisados separadamente: número de consultas de pré-natal, vacina antitetânica (se necessária), prescrição de sulfato ferroso, procedimentos de exame físico, orientações educativas recebidas e exames laboratoriais realizados. Para o exame físico, perguntou-se sobre a realização de cinco procedimentos pelo menos uma vez durante as consultas: aferição da altura uterina e da pressão arterial, exame ginecológico, de mamas e da cavidade oral. Quatro orientações educativas foram avaliadas: alimentação e ganho de peso, amamentação exclusiva até os seis meses, cuidados com o bebê e importância do exame preventivo do câncer do colo de útero. Perguntou-se também à mulher se ela havia realizado os seguintes exames pelo menos uma vez durante a gestação: comum de urina, glicemia, anti-HIV, detecção de sífilis (VDRL) e ultrassonografia. Os indicadores usados no presente estudo são todos os disponíveis no instrumento de avaliação externa do I Ciclo do PMAQ-AB 20. O referido instrumento foi validado pelas áreas técnicas do Ministério da Saúde e fazem parte do preconizado pelo protocolo do Ministério para pré-natal de baixo risco 7 .

Dessa forma, o pré-natal foi considerado com qualidade adequada quando a mulher afirmou ter realizado no mínimo seis consultas durante a gestação e todos os cinco exames complementares, bem como ter feito vacina antitetânica (se necessário) e recebido prescrição de sulfato ferroso, ter recebido todos os cinco procedimentos do exame físico e as quatro orientações.

Cada um desses seis desfechos foram analisados separadamente e também em conjunto, usandose uma variável sintética. A escolha desses indicadores foi pautada pela disponibilidade no instrumento da pesquisa e por sua relevância para a saúde materna e infantil.

As variáveis individuais avaliadas foram: idade da mulher em anos completos (16-20; 21-24; 25-34 e 35-49), cor da pele autorreferida (mestiça/parda/preta; branca; amarela/indígena) e quartis de renda familiar mensal per capita em reais (até 104; 105-187; 188-300; 301 e mais).

Como variáveis de contexto, utilizou-se as regiões geopolíticas (Norte, Nordeste, Centro-oeste, Sudeste e Sul); o porte do município, em habitantes (até 10.000; 10.001-30.000; 30.001-100.000; 100.001-300.000; mais de 300.000); o Índice de Desenvolvimento Humano Municipal (IDH-M) 21 classificado em quartis $(0,467-0,642 ; 0,643-0,730 ; 0,731-0,787 ; 0,788-0,919)$ e a cobertura populacional da ESF em cada município (até 50\%; 50,1\%-75\%; 75,1\%-99,9\%; 100\%).

Os dados foram coletados por meio de formulários eletrônicos utilizando-se tablets com envio automatizado ao Ministério da Saúde. A análise de consistência do banco de dados ficou a cargo de cada uma das seis instituições que lideraram a coleta, sob a coordenação do Departamento de Atenção Básica do Ministério da Saúde.

O pacote estatístico Stata 12.0 (StataCorp LP, College Station, Estados Unidos) foi utilizado na análise dos dados. Além da análise descritiva, a prevalência da adequação dos desfechos foi calculada de acordo com as características sociodemográficas e de contexto. A análise dos fatores associados foi realizada usando-se o teste do qui-quadrado para heterogeneidade (variáveis nominais) e teste de tendência linear (variáveis ordinais).

O estudo foi aprovado pelo Comitê de Ética em Pesquisa da Faculdade de Medicina da UFPel, sob o Ofício 38/12 de 10 de maio de 2012. Todos os participantes assinaram o Termo de Consentimento Livre e Esclarecido. Os autores declaram não haver conflitos de interesse em relação ao tema de estudo. 


\section{Resultados}

De 50.791 mulheres incluídas na avaliação do PMAQ-AB em todo o território nacional, 45.560 $(89,8 \%)$ já haviam engravidado e 8.777 (19,3\%) tinham filhos com menos de dois anos de idade; destas, $8.667(98,9 \%)$ realizaram o pré-natal na última gravidez e $6.125(70,7 \%)$ o fizeram em 4.924 UBS onde estavam sendo entrevistadas. Os dados dessas 6.125 mulheres foram analisados no presente artigo.

Dessas mulheres, 43,6\% encontravam-se na faixa etária de 25 a 34 anos e 20\% na de 16 a 20 anos. Aquelas que tinham a pele mestiça, parda e preta foram maioria no estudo, totalizando 63,6\%. Em relação à renda familiar per capita, cerca de um quarto das entrevistadas recebia até $R \$ 104,00$, e 22,5\% recebiam R\$301,00 ou mais (Tabela 1).

\section{Tabela 1}

Distribuição das usuárias que tiveram filho nos últimos dois anos e que fizeram pré-natal nas unidades básicas de saúde (UBS) estudadas. Programa de Melhoria do Acesso e da Qualidade (PMAQ-AB), Brasil, 2012.

\begin{tabular}{|c|c|c|}
\hline Variável & $\mathbf{n}$ & $\%$ \\
\hline \multicolumn{3}{|l|}{ Idade (anos) [n = 6.024] } \\
\hline $16-20$ & 1.202 & 20,0 \\
\hline $21-24$ & 1.486 & 24,6 \\
\hline $25-34$ & 2.627 & 43,6 \\
\hline $35-49$ & 709 & 11,8 \\
\hline \multicolumn{3}{|l|}{ Cor da pele $[n=6.094]$} \\
\hline Mestiça/Parda/Preta/Outra & 4.111 & 67,5 \\
\hline Branca & 1.983 & 32,5 \\
\hline \multicolumn{3}{|c|}{ Renda familiar per capita (Reais) $[\mathrm{n}=5.022]$} \\
\hline Até 104 & 1.257 & 25,0 \\
\hline $105-187$ & 1.265 & 25,2 \\
\hline $188-300$ & 1.372 & 27,3 \\
\hline 301 e mais & 1.128 & 22,5 \\
\hline \multicolumn{3}{|l|}{ Região [n = 6.017] } \\
\hline Norte & 337 & 5,6 \\
\hline Nordeste & 2.002 & 33,3 \\
\hline Sudeste & 2.568 & 42,7 \\
\hline Sul & 545 & 9,0 \\
\hline Centro-oeste & 565 & 9,4 \\
\hline \multicolumn{3}{|c|}{ Porte do município (habitantes) [n = 6.017] } \\
\hline Até 10.000 & 958 & 15,9 \\
\hline $10.001-30.000$ & 1.773 & 29,5 \\
\hline $30.001-100.000$ & 1.253 & 20,8 \\
\hline $100.001-300.000$ & 734 & 12,2 \\
\hline Mais de 300.000 & 1.299 & 21,6 \\
\hline \multicolumn{3}{|l|}{ IDH (quartis) [n = 5.899] } \\
\hline $0,467-0,342$ & 1.273 & 21,6 \\
\hline $0,643-0,730$ & 1.400 & 23,7 \\
\hline $0,731-0,787$ & 1.362 & 23,1 \\
\hline $0,788-0,919$ & 1.864 & 31,6 \\
\hline \multicolumn{3}{|c|}{ Cobertura de saúde da família (\%) [n = 6.012] } \\
\hline Até 50 & 1.656 & 27,5 \\
\hline $50,1-75,0$ & 1.486 & 24,7 \\
\hline $75,1-99,9$ & 1.164 & 19,4 \\
\hline 100,0 & 1.707 & 28,4 \\
\hline
\end{tabular}

IDH: Índice de Desenvolvimento Humano. 
As regiões Sudeste e Nordeste concentraram a maioria das mulheres entrevistadas (76\%) (Tabela 1). Quase metade das mulheres $(45,4 \%)$ vivia em municípios com até 30 mil habitantes e com IDH-M inferior a 0,731 (45,3\%). Menos de 30\% das entrevistadas residiam em municípios com baixa cobertura da $\operatorname{ESF}(27,5 \%)$ e 28,4\% em municípios com 100\% de cobertura (Tabela 1 ).

\section{Desfechos}

Quase 100\% das mulheres referiram ter atualizado sua vacina antitetânica e recebido prescrição de sulfato ferroso, além de $89 \%$ delas terem realizado seis ou mais consultas durante a gestação (Figura 1).

Em relação ao exame físico, menos de um quarto das entrevistadas (23,6\%) referiu ter recebido todos os procedimentos investigados durante o pré-natal da última gestação, sendo a aferição da pressão arterial e da altura uterina os mais frequentes e o exame ginecológico o menos frequente (Figura 1).

A proporção de mulheres que recebeu todas as orientações foi de $60,3 \%$, sendo as orientações sobre amamentação exclusiva a mais oferecida, e sobre a importância do pré-câncer a menos oferecida (Figura 1).

Por fim, 69,2\% das entrevistadas afirmaram ter realizado todos os exames complementares durante o pré-natal, sendo o VDRL e a glicemia os exames com menor prevalência, e o comum de urina, anti-HIV e ultrassonografia registraram maior prevalência (Figura 1).

\section{Figura 1}

Prevalência de procedimentos, exames e orientações recebidos durante o pré-natal na rede básica de saúde. Programa de Melhoria do Acesso e da Qualidade (PMAQ-AB), Brasil, 2012.

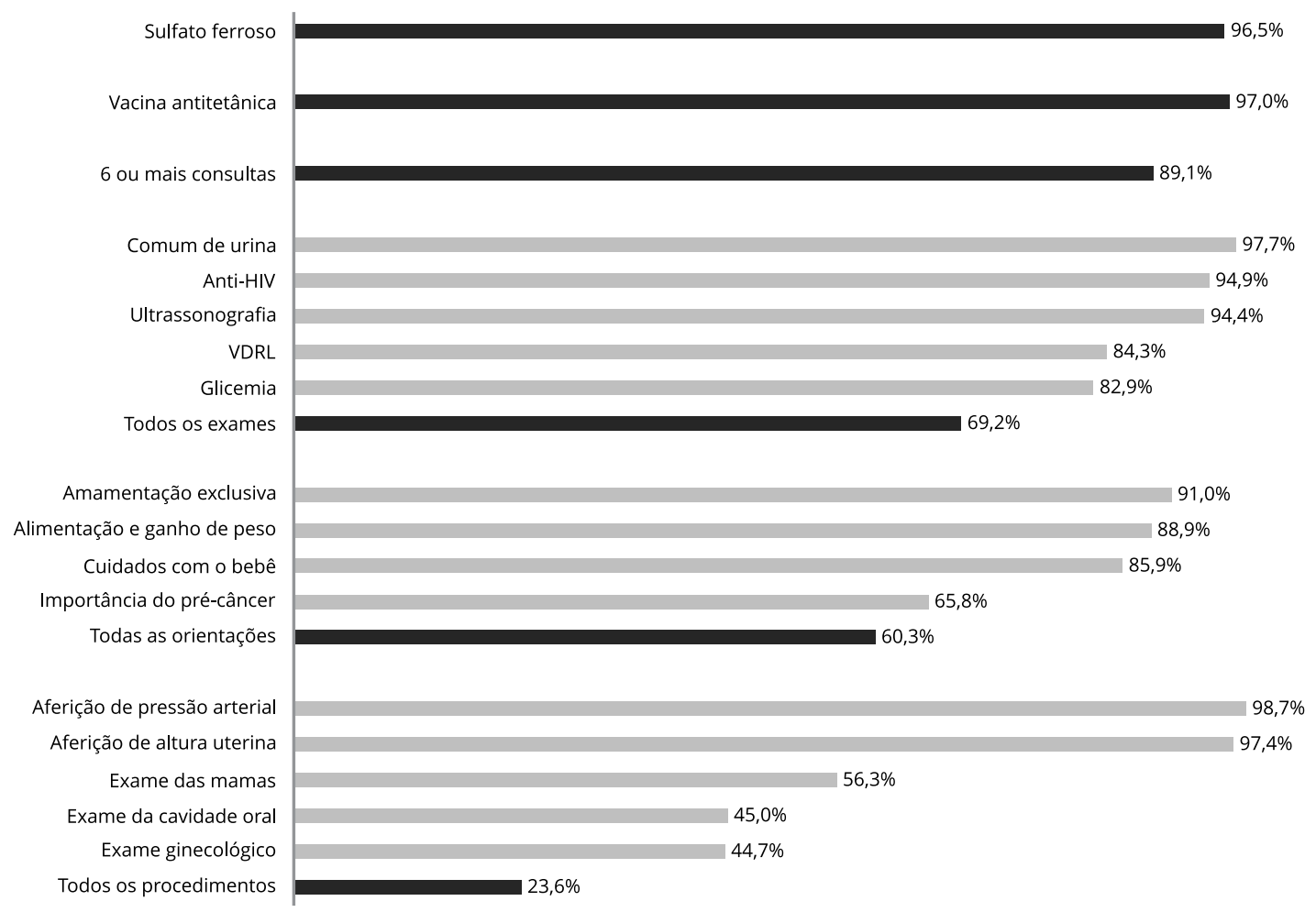




\section{Associações}

A proporção de mulheres que realizaram seis ou mais consultas durante a gestação foi maior entre as de mais idade, as brancas, as de renda mais alta, moradoras na Região Sul e em municípios de menor porte.

A prescrição de sulfato ferroso foi significativamente maior entre as mulheres não brancas, as mais pobres, as da Região Nordeste, residentes em municípios com menor IDH e com maior cobertura de saúde da família (Tabela 2).

A atualização da vacina antitetânica foi significativamente maior entre as de mais idade, as não brancas, as residentes em municípios de médio porte e com menor IDH.

Não houve diferença para o exame físico de acordo com as variáveis cor da pele e renda familiar. Entretanto, observou-se que quanto maior a idade, significativamente maiores foram as proporções de exame físico completo (Tabela 2). As regiões Centro-oeste e Norte foram as que registraram as menores prevalências de completude de exame físico (11,9\% e 17,2\%, respectivamente) e a Sudeste registrou a maior (28\%). Em relação ao porte populacional, nos municípios muito grandes, 30,3\% das mulheres realizaram o exame completo, sendo que os demais apresentaram proporções inferiores e similares. O mesmo comportamento foi observado para o IDH-M. Não houve diferenças significativas conforme a cobertura municipal de saúde da família (Tabela 2).

O fornecimento de orientações esteve positivamente associado com a idade, isto é, quanto maior a faixa etária, maiores as proporções de orientações recebidas. Mulheres de mais baixa renda receberam menos orientações durante o pré-natal (Tabela 2). As regiões Centro-oeste e Norte apresentaram as menores proporções de orientações completas (45,1\% e 54,9\%, respectivamente) e a Sudeste a maior $(63,4 \%)$. Quanto maior o porte do município, maiores as prevalências de orientações fornecidas às gestantes durante o pré-natal $(\mathrm{p}=0,018)$, tendo sido observado também que as gestantes residentes em municípios de IDH muito alto receberam mais orientações (64,3\%). Mulheres residentes em municípios com menor cobertura de saúde da família receberam mais orientações (Tabela 2).

A realização de todos os exames complementares aumentou com a idade e a renda familiar, ambos com $\mathrm{p}<0,001$. A totalidade dos exames foi realizada em maior proporção por mulheres brancas do que pelas demais ( $<$ 0,001) (Tabela 2). Na Região Centro-oeste e nos dois quartis superiores de IDH foi obtida a maior prevalência de exames complementares realizados (cerca de 75\%), não tendo sido significativamente diferentes as prevalências de acordo com o tamanho do município e nem com a sua cobertura de saúde de família (Tabela 2).

A análise do comportamento do indicador sintético de qualidade do pré-natal mostrou que 15\% das gestantes receberam atenção com qualidade adequada, e a pior atenção pré-natal foi dedicada às mulheres mais jovens, de menor renda familiar, das regiões Norte e Centro-oeste, de municípios com menor porte e com menor IDH (Tabela 2).

\section{Discussão}

Este é o primeiro trabalho dedicado a avaliar aspectos da qualidade da atenção pré-natal prestada na rede básica de saúde em todo o Brasil, e a investigar possíveis iniquidades sociais. De acordo com os parâmetros utilizados, os achados apontam para uma situação preocupante, uma vez que apenas $15 \%$ das entrevistadas receberam uma atenção de qualidade, percentual inferior ao encontrado no recente estudo nacional de base hospitalar 22 , que registrou $22 \%$ de adequação considerando parâmetros similares.

Menos de um quarto das gestantes realizou exame físico apropriado e pouco mais da metade receberam todas as orientações preconizadas. Considerando que tais ações dependem quase que exclusivamente da atitude dos profissionais de saúde, o desafio parece ser o de compreender os motivos pelos quais essas ações não estão sendo integralmente disponibilizadas à população-alvo. Os exames de mama, ginecológico e da cavidade oral foram ofertados a cerca de 50\% das gestantes. A falta dos exames da cavidade oral pode estar relacionada com a cobertura muito mais baixa de equipes de saúde bucal quando comparada com a de médicos e enfermeiros. Também pode ser reflexo de ideias equivocadas, tais como a de que médicos e enfermeiros não teriam essa atribuição de avaliar as necessidades 
Tabela 2

Distribuição dos seis desfechos investigados e qualidade adequada no pré-natal de acordo com as características individuais, região, porte, Índice de Desenvolvimento Humano (IDH) e cobertura de saúde da família dos municípios. Programa de Melhoria do Acesso e da Qualidade (PMAQ-AB), Brasil, 2012.

\begin{tabular}{|c|c|c|c|c|c|c|c|}
\hline Variável & $\begin{array}{c}6 \text { ou mais } \\
\text { consultas (\%) }\end{array}$ & $\begin{array}{c}\text { Sulfato } \\
\text { ferrosos (\%) }\end{array}$ & $\begin{array}{c}\text { Vacina } \\
\text { antitetânica (\%) }\end{array}$ & $\begin{array}{l}\text { Exame } \\
\text { físico (\%) }\end{array}$ & $\begin{array}{c}\text { Orientações } \\
(\%)\end{array}$ & $\begin{array}{c}\text { Exames } \\
\text { complementares (\%) }\end{array}$ & $\begin{array}{c}\text { Pré-natal com } \\
\text { qualidade } \\
\text { adequada * }(\%)\end{array}$ \\
\hline Idade (anos) & $<0,001 * \star$ & $0,661 * *$ & $<0,001 * *$ & $<0,001 * *$ & $<0,001 * *$ & $<0,001 * *$ & $<0,001 * \star$ \\
\hline $16-20$ & 85,2 & 97,3 & 94,8 & 17,8 & 51,6 & 60,4 & 10,0 \\
\hline $21-24$ & 89,9 & 95,8 & 96,8 & 20,1 & 59,0 & 64,7 & 12,2 \\
\hline $25-34$ & 90,6 & 96,5 & 97,7 & 25,9 & 62,9 & 73,8 & 17,4 \\
\hline $35-49$ & 88,8 & 96,7 & 98,6 & 32,7 & 69,3 & 77,3 & 19,9 \\
\hline Cor da pele & $<0,05 * * *$ & $<0,05 * * *$ & $<0,05 * * *$ & $0,712 * * *$ & $0,570 * * *$ & $<0,001 * * *$ & $<0,05 * * *$ \\
\hline $\begin{array}{l}\text { Mestiça/Parda/ } \\
\text { Preta/Outra }\end{array}$ & 88,3 & 97,0 & 97,3 & 23,4 & 60,1 & 67,2 & 14,9 \\
\hline Branca & 90,8 & 95,5 & 96,3 & 23,9 & 60,9 & 73,8 & 15,3 \\
\hline $\begin{array}{l}\text { Renda familiar per } \\
\text { capita (Reais) }\end{array}$ & $<0,001 * *$ & $<0,05 * *$ & 0,265 ** & 0,457 ** & 0,056 ** & $<0,001$ ** & $<0,05 * *$ \\
\hline Até 104 & 86,8 & 97,3 & 97,3 & 22,9 & 58,8 & 61,5 & 13,6 \\
\hline $105-187$ & 88,4 & 96,4 & 97,5 & 23,1 & 60,7 & 68,6 & 14,2 \\
\hline $188-300$ & 91,4 & 96,3 & 96,6 & 24,4 & 60,7 & 72,7 & 16,4 \\
\hline 301 e mais & 91,0 & 94,8 & 96,8 & 23,8 & 62,9 & 76,4 & 16,0 \\
\hline Região & $<0,001 * \star *$ & $<0,001 * * *$ & $0,133 * * *$ & $<0,001 * * *$ & $<0,001 * * *$ & $<0,001 * * *$ & $<0,001 * * *$ \\
\hline Norte & 81,5 & 94,9 & 95,8 & 17,2 & 54,9 & 55,5 & 8,0 \\
\hline Nordeste & 88,6 & 98,2 & 97,9 & 22,9 & 61,3 & 66,4 & 14,9 \\
\hline Sudeste & 89,7 & 96,6 & 96,8 & 28,0 & 63,4 & 71,2 & 18,0 \\
\hline Sul & 93,0 & 92,4 & 96,3 & 20,9 & 61,7 & 73,6 & 12,7 \\
\hline Centro-oeste & 88,1 & 95,2 & 96,5 & 11,9 & 45,1 & 75,2 & 7,6 \\
\hline $\begin{array}{l}\text { Porte do município } \\
\text { (habitantes) }\end{array}$ & 0,010 ** & $0,778 * *$ & $<0,05 * *$ & $0,001 * *$ & 0,018 ** & $0,832 * *$ & $<0,05 * *$ \\
\hline Até 10.000 & 90,1 & 95,7 & 96,8 & 22,0 & 58,4 & 69,1 & 15,0 \\
\hline $10.001-30.000$ & 89,6 & 96,9 & 98,1 & 21,4 & 59,4 & 69,7 & 13,3 \\
\hline $30.001-100.000$ & 89,7 & 97,4 & 97,0 & 21,5 & 60,7 & 69,0 & 14,7 \\
\hline $100.001-300.000$ & 89,9 & 96,2 & 96,2 & 22,1 & 59,9 & 70,4 & 14,6 \\
\hline Mais de 300.000 & 86,5 & 96,1 & 96,2 & 30,3 & 63,1 & 68,7 & 17,7 \\
\hline IDH (quartis) & $0,993 * *$ & $<0,001 * *$ & $0,05 * *$ & $<0,001 * *$ & $0,018 * *$ & $<0,001 * *$ & $<0,05 * *$ \\
\hline $0,467-0,342$ & 89,4 & 98,1 & 98,2 & 21,8 & 60,3 & 63,2 & 13,7 \\
\hline $0,643-0,730$ & 88,5 & 96,7 & 96,6 & 21,9 & 58,6 & 66,1 & 14,1 \\
\hline $0,731-0,787$ & 89,3 & 96,0 & 97,4 & 20,9 & 57,5 & 73,2 & 13,3 \\
\hline $0,788-0,919$ & 89,1 & 95,7 & 96,2 & 28,5 & 64,3 & 72,9 & 18,0 \\
\hline $\begin{array}{l}\text { Cobertura de saúde } \\
\text { da família (\%) }\end{array}$ & $0,401 * *$ & $<0,05 * *$ & 0,092 ** & $0,235 * *$ & $0,061 * *$ & 0,578 ** & 0,535 ** \\
\hline Até 50 & 88,8 & 95,8 & 97,0 & 25,5 & 63,6 & 68,5 & 15,7 \\
\hline $50,1-75,0$ & 88,4 & 96,8 & 96,3 & 22,4 & 59,2 & 71,9 & 13,5 \\
\hline $75,1-99,9$ & 89,7 & 96,1 & 96,8 & 22,3 & 57,1 & 68,1 & 13,9 \\
\hline 100,0 & 89,4 & 97,3 & 97,8 & 23,6 & 60,6 & 68,7 & 16,3 \\
\hline Total & 89,1 & 96,5 & 97,0 & 23,6 & 60,3 & 69,2 & 15,0 \\
\hline
\end{tabular}

* Mulher afirmou ter recebido todos os cinco procedimentos do exame físico, as quatro orientações, realizado todos os cinco exames, feito seis ou mais consultas, atualizado vacina antitetânica e recebido prescrição de sulfato ferroso;

** Valor de $p$ de tendência linear;

$\star * \star$ Valor de $\mathrm{p}$ de heterogeneidade. 
em saúde bucal, bem como a de que não adiantaria identificar problemas de saúde bucal porque não é possível encaminhar ou resolver.

Possíveis deficiências na estrutura das UBS também podem ter concorrido para as baixas prevalências de realização de exames ginecológico e de mama 23 , como a falta de materiais e insumos, entre eles luvas, focos, lâminas ou fixadores. Dados do censo das UBS realizado em 2012 revelaram que 17\% delas não dispunham de mesa apropriada para esse exame e $8 \%$ não tinham mesa de exame clínico 24 , mas nas UBS onde essas mulheres realizaram o pré-natal não havia mesa com perneiras em apenas $5 \%$ dos casos. Além disso, acredita-se que os processos de trabalho possam ser revistos, especialmente no que diz respeito às atribuições dos profissionais expressas pelo protocolo do Ministério da Saúde, que inclui esse tipo de exame entre as funções de médicos e enfermeiros 7.

Em relação ao fornecimento de orientações, tais ações não implicam custos para o SUS, sendo unicamente dependentes das atitudes dos profissionais. Quais seriam os fatores que inviabilizam a realização dessas ações? Escassa formação dos profissionais de saúde para fazer educação em saúde, afetando não somente o baixo fornecimento, mas também a qualidade das orientações prestadas? Falta de tempo durante a jornada de trabalho? O ritmo de produção de atendimentos cobrado pela gestão impõe um padrão de qualidade reduzido?

Um dos achados mais animadores foi que, entre as variáveis individuais analisadas, a cor da pele não esteve associada à qualidade do exame físico e das orientações, tampouco ao indicador sintético global, o que reforça a equidade na dispensação dessas ações. Esses resultados vão de encontro do que foi evidenciado por diferentes estudos 10,14,15,16, provavelmente pela relativa homogeneidade socioeconômica das mulheres em nossa amostra, uma vez que entraram no estudo por estarem vinculadas a UBS localizadas, em sua maioria, a áreas de maior vulnerabilidade social. Nos projetos de implantação da ESF nos municípios, um dos critérios adotados pela gestão para a escolha das unidades foi a sua localização em áreas de maior vulnerabilidade, atendendo as diretrizes da Política Nacional de Atenção Básica (PNAB).

Entretanto, os dados parecem apontar para uma grave iniquidade relacionada à idade das gestantes, pois sistematicamente as adolescentes ficaram com os mais baixos índices, tanto nos desfechos isolados - exame físico, orientações e exames complementares - quanto no indicador sintético. Esses achados são similares aos descritos por Gama et al. 25 e por Domingues et al. 22. O agravante diz respeito ao fato de que a gestação na adolescência apresenta maior risco, tanto para a gestante quanto para o recém-nascido, assim, o pré-natal inadequado neste grupo indica que o serviço não está priorizando um grupo com grandes necessidades em saúde 26,27. Outro fator que pode estar associado a esse resultado é o menor acesso de adolescentes aos serviços de saúde de um modo geral 28, reflexo da escassez de políticas públicas voltadas aos adolescentes, que pode interferir na qualidade do serviço prestado. Além disso, mulheres com mais idade, pela possibilidade de estarem mais expostas ao cuidado prénatal em gestações prévias ou por meio de outras fontes, podem lembrar mais das recomendações do que as mais jovens.

Diferenças significativas também foram observadas ao se analisar a realização de exames complementares. Como a UBS é responsável apenas pela solicitação dos exames, sua realização pode ter sido condicionada ao maior poder aquisitivo e menor vulnerabilidade racial, pois as mulheres de cor branca e aquelas de maior renda familiar referiram significativamente ter feito mais exames do que as demais 29,30 .

Em relação às variáveis de contexto, fortes associações foram registradas para todos os desfechos e a região geopolítica, com consistentes piores índices para as regiões Norte, Nordeste e Centro-oeste, reforçando as já conhecidas desigualdades sociais regionais vigentes em nosso país 31,32.

Com exceção da realização de exames e da prescrição de sulfato ferroso, as análises dos demais desfechos segundo o porte do município mostraram que os maiores conseguem prestar uma atenção pré-natal de melhor qualidade do que os menores, mas ainda assim ficando longe do esperado. Embora os municípios pequenos tenham maior cobertura de ESF, eles têm menor rede para exames complementares, muitas vezes dependendo da regionalização para garantir a oferta destes exames. O fato de eventualmente implicar deslocamento para municípios vizinhos também dificulta a realização dos exames.

Observando-se as associações dos desfechos com o IDH-M, curiosamente a vacina antitetânica e o sulfato ferroso registraram melhores prevalências em municípios no quartil mais baixo, e os indica- 
dores de exame físico, orientações e exames complementares apresentaram tendência linear crescente com o aumento do índice, provavelmente por uma melhor estrutura e presença de equipe completa nas unidades de saúde.

Outro achado interessante foi a falta de associação de todos os desfechos com a cobertura de saúde da família dos municípios, com exceção da prescrição de sulfato ferroso. De modo geral, as coberturas tendem a ser maiores em municípios menores e mais pobres, em função das características da implantação e da expansão da estratégia no território nacional ao longo das últimas duas décadas.

Assim, seria esperado que padrão contrário ao do IDH fosse observado, e que quanto mais ESF, mais qualidade na atenção às gestantes, mas as diferenças não apresentaram nenhum padrão nem foram estatisticamente significativas. Ao analisar o desfecho de qualidade global com a cobertura de Saúde da Família estratificando para o IDH, observou-se que, com exceção dos municípios com o índice mais alto, nos demais estratos maiores coberturas de Saúde da Família implicaram melhor qualidade. Provavelmente, o fato do pré-natal ser uma das ações programáticas de maior capilaridade no âmbito da atenção primária à saúde, outros fatores relacionados à formação dos profissionais e à organização e gestão dos serviços - mais homogêneos - tenham pesado mais no exame destas associações do que a heterogênea cobertura municipal da ESF.

Antes das entrevistas, as equipes de saúde das UBS sabiam que faziam parte de um processo avaliativo e poderiam ter instigado seus usuários a minimizarem os problemas e enfatizarem os aspectos positivos. A amostra foi unicamente de usuárias dos serviços, o que pode tê-las levado a referir recebimento de mais orientações e procedimentos do que a realidade, mesmo assim, as prevalências dos desfechos foram baixas; se houve superestimativa, mais preocupantes tornam-se os achados. Por ter sido restrito à atenção primária, não foi possível comparar com outros níveis de atenção; para o estabelecimento de padrões foram utilizadas as recomendações dos protocolos do Ministério da Saúde.

Em favor da redução das desigualdades, sugere-se que o tema da qualidade da atenção pré-natal na rede básica de saúde em nosso país continue a ser estudado em novas pesquisas, notadamente por meio de estudos de intervenção, de modo a testar a efetividade de ações de qualificação das equipes e dos processos de trabalho.

\section{Colaboradores}

E. Tomasi participou da concepção do artigo, análise e interpretação dos dados e redação do artigo. P. A. A. Fernandes e T. Fischer colaboraram na análise e interpretação dos dados e revisão do texto. E. Thumé, D. S. Silveira, F. C. V. Siqueira, S. M. S. Duro, M. O. Saes, B. P. Nunes e A. G. Fassa contribuíram na revisão crítica relevante do conteúdo intelectual e aprovação final da versão a ser publicada. L. A. Facchini colaborou na concepção do artigo, revisão crítica relevante do conteúdo intelectual e aprovação final da versão a ser publicada. Todos os autores são responsáveis por todos os aspectos do trabalho na garantia da exatidão e integridade de qualquer parte da obra.

\section{Agradecimentos}

Ao Departamento de Atenção Básica da Secretaria de Ações de Saúde do Ministério da Saúde pelo financiamento.

\section{Referências}

1. Departamento de Articulação Interfederativa, Secretaria de Gestão Estratégica e Participativa, Ministério da Saúde. Caderno de diretrizes, objetivos, metas e indicadores 2013-2015. Brasília: Ministério da Saúde; 2014. (Série Articulação Interfederativa, 1).

2. Ministério da Saúde. Pré-natal e puerpério: atenção qualificada e humanizada: manual técnico. Brasília: Ministério da Saúde; 2005.

3. Barbeiro FMS, Fonseca SC, Tauffer MG, Ferreira MSS, Silva FP, Ventura PV, et al. Óbitos fetais no Brasil: revisão sistemática. Rev Saúde Pública 2015; 49:22.

4. Lansky S, Friche AAL, Silva AAM, Campos D, Bittencourt SDA, Carvalho ML, et al. Pesquisa Nascer no Brasil: perfil da mortalidade neonatal e avaliação da assistência à gestante e ao recémnascido. Cad Saúde Pública 2014; 30 Suppl 1: S192-207.

5. Oyerinde K. Can antenatal care result in significant maternal mortality reduction in developing countries? J Community Med Health Educ 2013; 3:e116. 
6. Hong R, Ruiz-Beltran M. Impact of prenatal care on infant survival in Bangladesh. Matern Child Health J 2007; 11:199-206.

7. Departamento de Atenção Básica, Ministério da Saúde. Atenção ao pré-natal de baixo risco. Brasília: Ministério da Saúde; 2012.

8. Hill Z, Kirkwood BR, Edmond K. Family and community practices that promote child survival, growth and development: a review of the evidence. Geneva: World Health Organization; 2004.

9. Rasia ICRB, Albernaz E. Atenção pré-natal na cidade de Pelotas, Rio Grande do Sul, Brasil. Rev Bras Saúde Matern Infant 2008; 8:401-10.

10. Viellas EF, Domingues RMSM, Dias MAB, Gama SGN, Theme Filha MM, Costa JV, et al. Assistência pré-natal no Brasil. Cad Saúde Pública 2014; 30 Suppl 1:S85-100.

11. Silveira DS, Santos IS, Dias-da-Costa JS, Atenção pré-natal na rede básica: uma avaliação da estrutura e do processo. Cad Saúde Pública 2001; 17:131-9.

12. Anversa ETR, Bastos GAN, Nunes LN, Dal Pizzol TS. Qualidade do processo da assistência pré-natal: unidades básicas de saúde e unidades de Estratégia Saúde da Família em município no Sul do Brasil. Cad Saúde Pública 2012; 28:789-800.

13. Carvalho DS, Novaes HMD. Avaliação da implantação de programa de atenção pré-natal no Município de Curitiba, Paraná, Brasil: estudo em coorte de primigestas. Cad Saúde Pública 2004; 20 Suppl 2:S220-30.

14. Leal MC, Gama SGN, Cunha CB, Desigualdades raciais, sociodemográficas e na assistência ao pré-natal e ao parto, 1999-2001. Rev Saúde Pública 2005; 39:100-7.

15. Coimbra LC, Silva AAM, Mochel EG, Alves MTSSB, Ribeiro VS, Aragão VMF, et al. Fatores associados à inadequação do uso da assistência pré-natal. Rev Saúde Pública 2003; 37: 456-62.

16. Cesar JA, Mendoza-Sassi RA, Gonzalez-Chica DA, Mano PS, Goulart-Filha SM. Características sociodemográficas e de assistência à gestação e ao parto no extremo Sul do Brasil. Cad Saúde Pública 2011; 27:985-94.

17. Ministério da Saúde. Portaria no $1.654 / \mathrm{GM} /$ MS, de 19 de julho de 2011. Institui, no âmbito do Sistema Único de Saúde, o Programa Nacional de Melhoria do Acesso e da Qualidade da Atenção Básica (PMAQ-AB) e o Incentivo Financeiro do PMAQ-AB, denominado Componente de Qualidade do Piso de Atenção Básica Variável - PAB Variável. Diário Oficial da União 2011; 20 jul.

18. Departamento de Atenção Básica, Secretaria de Atenção à Saúde, Ministério da Saúde. Saúde Mais Perto de Você. Programa Nacional da Melhoria do Acesso e da Qualidade da Atenção Básica: manual instrutivo. Brasília: Ministério da Saúde; 2012.

19. Giovanella L, Bousquat A, Fausto MCR, Fusaro ER, Mendonça MHM, Gagno J. Novos caminhos: tipologia das unidades básicas de saúde brasileiras. Brasília: Região e Redes; 2015.
20. Ministério da Saúde. Instrumento de avaliação externa do Saúde Mais Perto de Você - acesso e qualidade. Programa Nacional de Melhoria do Acesso e da Qualidade da Atenção Básica (PMAQ). Brasília: Ministério da Saúde; 2012.

21. Programa das Nações Unidas para o Desenvolvimento. Índice de desenvolvimento humano, 2014. http://www.pnud.org.br/IDH/DH.aspx (acessado em 31/Jul/2015).

22. Domingues RMSM, Dias MAB, Leal MC, Gama SGN, Theme-Filha MM, Torres JA, et al. Adequação da assistência pré-natal segundo as características maternas no Brasil. Rev Panam Salud Pública 2015; 37:140-7.

23. Donabedian A. The quality of care. How can it be assessed? JAMA 1988; 260:1743-8.

24. Ministério da Saúde. Retratos da Atenção Básica no 2 - gestão da atenção básica. Volume 2 insumos e medicamentos nas unidades básicas de saúde. Brasília: Ministério da Saúde; 2015.

25. Gama SGN, Szwarcwald CL, Leal MC. Experiência de gravidez na adolescência, fatores associados e resultados perinatais entre puérperas de baixa renda. Cad Saúde Pública 2002; 18:153-61.

26. Gama SGN, Szwarcwald CL, Sabroza AR, Branco VC, Leal MC. Fatores associados à assistência pré-natal precária em uma amostra de puérperas adolescentes em maternidades do Município do Rio de Janeiro, 1999-2000. Cad Saúde Pública 2004; 20 Suppl 1:S101-1.

27. Sawyer SM, Afifi RA, Bearinger LH, Blakemore SJ, Dick B, Ezeh AC, et al. Adolescence: a foundation for future health. Lancet 2012; 379:1630-40.

28. Instituto Brasileiro de Geografia e Estatística. Pesquisa Nacional por Amostra de Domicílios. Um panorama da saúde no Brasil: acesso e utilização de serviços, condições de saúde e fatores de risco e proteção à saúde, 2008. Rio de Janeiro: Instituto Brasileiro de Geografia e Estatística; 2010.

29. Silva EP, Lima RT, Ferreira NLS, Costa MJC. Pré-natal na atenção primária do Município de João Pessoa - PB: caracterização de serviços e usuárias. Rev Bras Saúde Matern Infant 2013; 13:29-37.

30. Martinelli KG, Santos Neto ET, Gama SGN, Oliveira AE. Adequação do processo da assistência pré-natal segundo os critérios do Programa de Humanização do Pré-natal e Nascimento e Rede Cegonha. Rev Bras Ginecol Obstet $2014 ; 36: 56-64$.

31. Travassos C, Oliveira EXG, Viacava F. Desigualdades geográficas e sociais no acesso aos serviços de saúde no Brasil: 1998 e 2003. Ciênc Saúde Coletiva 2006; 11:975-86.

32. Victora CG, Barreto ML, Leal MC, Monteiro CA, Schmidt MI, Paim JS, et al. Health conditions and health-policy innovations in Brazil: the way forward. Lancet 2011; 377:2042-53. 


\section{Abstract}

The aim of this study was to describe quality indicators for prenatal care in Brazil as part of the Program for the Improvement of Access and Quality (PMAQ-AB). The study analyzed number of prenatal visits, vaccination status, prescription of ferrous sulfate, physical examination, orientation, and laboratory tests, based on which a summary quality indicator was constructed. Data were collected in 2012-2013 during interviews conducted by External Evaluators of the PMAQ-AB, with 6,125 users who had done their last prenatal follow-up in Family Health units. During prenatal follow-up, $89 \%$ reported six or more visits, more than $95 \%$ received a tetanus booster and prescription of ferrous sulfate, $24 \%$ reported having received all the procedures in the physical examination, $60 \%$ received all the orientation, and 69\% had all the recommended laboratory tests. Only 15\% of interviewees had received adequate prenatal care, including all the recommended measures, and there was a significantly higher proportion of "complete" care in pregnant women that were older, with higher income, in the Southeast region of Brazil, in municipalities with more than 300,000 inhabitants, and in those with HDI in the upper quartile. There are persist social and individual inequalities that can be targeted by measures to upgrade the teams' work processes.

Prenatal Care; Primary Health Care; Quality of Health Care; Health Inequalities

\section{Resumen}

El objetivo fue describir indicadores de calidad de la atención prenatal en Brasil en el ámbito del Programa de Mejoría del Acceso y de la Calidad (PMAQ-AB). Se analizaron el número de consultas, situación de vacunación, prescripción de sulfato ferroso, examen físico, orientaciones y exámenes complementarios, en base a lo que se construyó como un indicador sintético de calidad. Los datos fueron recogidos en 2012/2013, mediante entrevistas realizadas por evaluadores externos del PMAQ-AB, entre 6.125 usuarias que realizaron su último examen prenatal en las unidades de Salud de la Familia. Durante el periodo prenatal, un $89 \%$ realizaron seis o más consultas, más de un 95\% actualizaron la vacuna antitetánica y obtuvieron recetas de sulfato ferroso, un $24 \%$ informaron haber recibido todos los procedimientos de examen físico, un $60 \%$ recibieron todas las orientaciones y un $69 \%$ realizaron todos los exámenes complementarios. Solamente un 15\% de las entrevistadas recibieron atención prenatal adecuada, considerándose todas las acciones preconizadas, siendo significativamente mayor la proporción "de en su totalidad" en la atención en gestantes con más edad, de mayor renta, en la Región Sudeste, en los municipios con más de 300 mil habitantes y con IDH en el cuartil superior. Persisten desigualdades sociales e individuales que pueden ser objeto de acciones de cualificación de los procesos de trabajo de equipos de salud.

Atención Prenatal; Atención Primaria de Salud; Calidad de la Atención de Salud; Desigualdades en la Salud
Recebido em 01/Dez/2015

Versão final reapresentada em 06/Mar/2016

Aprovado em 02/Mai/2016 